\title{
Diagnóstico y manejo de la hipoglicemia en adultos diabéticos hospitalizados: evaluación de competencias en un equipo profesional multidisciplinario de salud
}

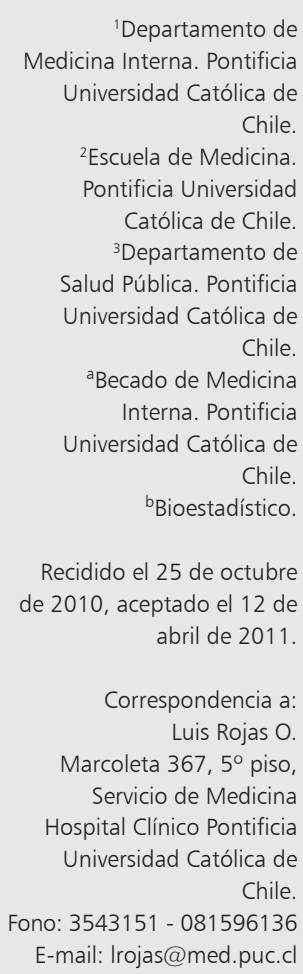

'Departamento de Medicina Interna. Pontificia Universidad Católica de Chile.

2Escuela de Medicina. Pontificia Universidad Católica de Chile. ${ }^{3}$ Departamento de Salud Pública. Pontificia Universidad Católica de Chile.

abecado de Medicina Interna. Pontificia Universidad Católica de Chile. ${ }^{\mathrm{b}}$ Bioestadístico.

Recidido el 25 de octubre de 2010, aceptado el 12 de abril de 2011.

Correspondencia a: Luis Rojas $\mathrm{O}$.

Marcoleta $367,5^{\circ}$ piso, Servicio de Medicina Hospital Clínico Pontificia Universidad Católica de Chile.

Fono: 3543151 - 081596136 E-mail: Irojas@med.puc.cl

\author{
LUIS ROJAS ${ }^{1}$, PABLO ACHURRA ${ }^{2}$, FELIPE PINO², PEDRO RAMÍREZ ${ }^{2}$, \\ MARCELO LOPETEGUI ${ }^{2}$, LUIS MANUEL SANHUEZA A. ${ }^{\text {a }}$ \\ LUIS VILLARROEL ${ }^{3, \mathrm{~b}}$, ANDRÉS AIZMAN ${ }^{1}$
}

\section{Competences on hypoglycemia management among healthcare professionals in a clinical hospital}

Background: A tight glycemic control of hospitalized patients increases the risk of hypoglycemia, whose management is not always optimal. Aim: To assess the hypoglycemia management competences of a multidisciplinary team in a clinical hospital. Material and Methods: An anonymous questionnaire about hypoglycemia management was answered by 11 staff physicians, 42 residents and 28 nurses of the department of medicine and critical care unit of a university hospital. Results: Respondents had a mean of $60 \%$ of correct answers, without significant differences between groups. The capillary blood glucose level that defines hypoglycemia was known by most of the respondents, but the value that defines severe episodes was known only by $60 \%$. The initial management and follow up was well known only for severe episodes. Less than 50\% knew the blood glucose value that required continuing with treatment. Conclusions: Although most professionals are able to recognize hypoglycemia, the knowledge about is management if insufficient.

(Rev Med Chile 2011; 139: 848-855).

Key words: Emergency medical services; Hypoglicemia; Patient care.
L a Diabetes Mellitus tipo 2 es una comorbilidad frecuente en pacientes hospitalizados, estando presente en alrededor de $25 \%$ de los casos. En estos pacientes la hiperglicemia se asocia a un aumento de la morbimortalidad, tiempo de estadía y $\operatorname{costos}^{1-7}$, por lo que el control glicémico es una importante meta a alcanzar en estos pacientes. Sin embargo, el cumplimiento de este objetivo se ve amenazada por el temor a una de sus principales complicaciones, la hipoglicemia ${ }^{8}$.

La hipoglicemia, definida para el paciente hospitalizado como leve cuando la glicemia capilar esta entre 40 y $69 \mathrm{mg} / \mathrm{dl}$ y severa cuando es menor a $40 \mathrm{mg} / \mathrm{dl}^{9}$, puede ocurrir tanto en pacientes no diabéticos (en cuyo caso es reflejo de la gravedad de la enfermedad concomitante ${ }^{10-12}$ ) como diabéticos, siendo los factores de riesgo más frecuentes la edad avanzada, malnutrición, cáncer activo, insuficiencia renal, enfermedad hepática e insuficiencia cardiaca congestiva ${ }^{13,14}$. La mayoría de las hipoglicemias son secundarias a fallas en el ajuste de las dosis de insulinas o al uso de hipoglicemiantes orales cuando la ingesta alimentaria disminuye $\mathrm{e}^{15-17}$ o cuando la causa que generó la hiperglicemia (por ejemplo infecciones) se resuelve y no se acompaña de un ajuste del tratamiento hipoglicemiante. Esto ha llevado a la Joint Commission on Accreditation of Healthcare a considerar estos fármacos dentro de los más peligrosos ${ }^{18}$.

La incidencia de hipoglicemia en pacientes 
diabéticos hospitalizados que reciben tratamiento es cercana al 9,5\% y en más del $40 \%$ de los casos presentan recurrencia del evento. Su impacto clínico está en discusión. Kosiborod determinó en un estudio retrospectivo que sólo aquellas hipoglicemias que ocurrían en forma espontánea se asociaban a mayor mortalidad y no las inducidas por insulina ${ }^{19}$. En contraposición a ello, Van Der Berghe, encontró una asociación entre hipoglicemias severas y aumento de la mortalidad, sin embargo, no entregó mayores detalles ${ }^{20}$.

Los pilares fundamentales en su enfrentamiento son la prevención ${ }^{21}$ y la terapia. Esta última debe considerar: 1) Evitar aportes indebidos de glucosa que causen marcadas fluctuaciones de glicemia; 2) Conductas que eviten la recurrencia, lo cual implica un seguimiento adecuado y pesquisa de los factores causales. Estos puntos son críticos ya que es donde se han reportado falencias importantes en estudios previos ${ }^{22}$.

Siendo un tema relevante para el cuidado de los pacientes hospitalizados, existen pocos estudios que permitan objetivar el nivel de conocimientos de los miembros del equipo de salud respecto al diagnóstico y manejo de la hipoglicemia ${ }^{23}$ y prácticamente ningún estudio ha evaluado el conocimiento de los médicos al respecto.

Por tal motivo, se realizó una evaluación del grado de conocimientos del equipo de salud (médicos de planta, médicos residentes y enfermeras) en un hospital docente respecto al diagnóstico y enfrentamiento clínico de los eventos hipoglicémicos leves y severos en pacientes adultos diabéticos hospitalizados.

\section{Hipótesis}

Se postula que existe insuficiente conocimiento acerca del diagnóstico y manejo de la hipoglicemia. Se cree también que se encontrarán diferencias entre los distintos estamentos, esperando que sean los médicos los mejor preparados.

\section{Objetivo principal}

Determinar el nivel de conocimiento de los distintos estamentos de un equipo de salud en el diagnóstico y manejo inmediato de la hipoglicemia en pacientes diabéticos hospitalizados.

\section{Objetivo secundario}

Determinar si existen diferencias en los niveles de conocimiento en los distintos tipos de hipoglicemias y en las etapas del manejo entre los diferentes estamentos del equipo de salud.

\section{Materiales y Métodos}

Se realizó un estudio observacional, descriptivo y transversal. Se aplicó un cuestionario anónimo a casi la totalidad de los médicos de planta, médicos residentes y enfermeras que trabajaran en las unidades de medicina y cirugía tanto de paciente crítico como no crítico del Hospital Clínico de la Universidad Católica de Chile entre febrero y mayo del año 2009.

El cuestionario se elaboró con preguntas relacionadas a aspectos diagnósticos, terapéuticos y de seguimiento basados en definiciones de la American Diabetes Association ${ }^{24}$ y en el protocolo de manejo de Hipoglicemia de Lovelance Medical Center $^{13}$. Cada pregunta tuvo 4 opciones de respuesta para evitar el sesgo de tendencia central (Anexo 1).

El cuestionario fue validado en dos aspectos: 1) El contenido: mediante cotejo entre referentes técnicos de los distintos estamentos profesionales de los servicios de hospitalización del Hospital Clínico de la Pontificia Universidad Católica de Chile (HCPUC) y 2) La forma: el cuestionario fue aplicado a 20 personas semejantes a la muestra que fue encuestada para así evaluar la comprensión de la redacción de las preguntas y el vocabulario usado.

El estudio contó con la aprobación y autorización de la Dirección del HCPUC y del Comité de Ética Local.

Las preguntas del cuestionario abarcaron situaciones de hipoglicemias según nivel de glicemia capilar y según presencia de sintomatología (asintomáticas, sintomáticas y con compromiso de conciencia). Los aspectos que fueron evaluados se agruparon en cinco áreas:

1. Diagnóstico de hipoglicemia y de su severidad.

2. Manejo terapéutico inicial según severidad y características del paciente (es decir, capacidad para recibir glucosa por vía enteral y presencia de vía venosa).

3. Tiempo en que debe realizarse una glicemia capilar de control (Seguimiento precoz).

4. Valor objetivo de glicemia capilar luego de la intervención inicial y que define las conductas a seguir.

5. Monitorización posterior a la obtención de este valor de glicemia capilar (seguimiento tardío). 
Una vez obtenidas las encuestas, se determinó el porcentaje de respuestas correctas para todo el grupo encuestado, para cada estamento, para cada tipo de hipoglicemia y para cada una de los ítems definidos

El análisis estadístico de las respuestas se realizó mediante test Anova para comparación de promedios entre los 3 grupos y se aplicó la prueba de $\chi^{2}$ de Pearson para la comparación de porcentajes para cada una de las preguntas entre los tres grupos descritos. Las diferencias se consideraron estadísticamente significativas con un valor de $\mathrm{p}<0,05$.

\section{Resultados}

Dicho cuestionario fue respondido por 11 médicos de planta (70\% del universo total), a 42 médicos residentes $(86 \%)$ y a 28 enfermeras $(72 \%)$ pertenecientes a los servicios de medicina y cirugía para pacientes críticos y no críticos del HCPUC.

\section{Rendimiento del grupo general}

El total de encuestados respondió correctamente el $60 \%$ de las preguntas, sin existir diferencias significativas entre los distintos estamentos. (Figura 1).

En relación al diagnóstico de hipoglicemia:

La definición operacional de hipoglicemia fue correctamente identificada por el $100 \%$ de los médicos de planta, el 92,8\% de los médicos residentes y por el $89,2 \%$ de las enfermeras. No hubo diferencias estadísticamente significativas entre los grupos $(\mathrm{p}=0,514)$.

La definición de hipoglicemia severa fue cono- cida correctamente por el 63,6\% de los médicos de planta, el $64,2 \%$ de los residentes y por el $67,8 \%$ de las enfermeras. Tampoco hubo diferencias significativas entre los grupos $(\mathrm{p}=0,945)$.

En relación a las conductas adoptadas en cada situación clínica:

El manejo inicial de la hipoglicemia fue respondido correctamente por el 70,8\% de los encuestados, siendo este porcentaje más alto en el escenario de hipoglicemias con compromiso de conciencia, en donde el porcentaje de respuestas correctas alcanzó el $85 \%$. El rendimiento entre los grupos sólo se diferenció a favor de los médicos de manera significativa en el escenario de las hipoglicemias sintomáticas no severas (Tabla 1).

En cuanto al seguimiento precoz, sólo el 50 a $60 \%$ de los encuestados conocía el lapso de tiempo en que debe realizarse una glicemia capilar de control. Al desglosar los resultados, el conocimiento fue mayor en el caso de las hipoglicemias con compromiso de conciencia y menor en las hipoglicemias asintomáticas (Tabla 1). El rendimiento fue parejo entre los estamentos.

En relación al conocimiento del valor de glicemia capilar objetivo después de la intervención inicial y que define las conductas a seguir, se encontraron respuestas correctas en menos del $50 \%$ de los encuestados (promedio: 44,4\%), siendo éstas más bajas para las hipoglicemias con compromiso de conciencia. Tampoco se objetivaron diferencias significativas entre los distintos estamentos.

Fue en relación a la monitorización posterior o seguimiento tardío (conductas que evitan la recurrencia del cuadro) en donde se obtuvo el menor porcentaje de respuestas correctas $(34,9 \%)$, siendo

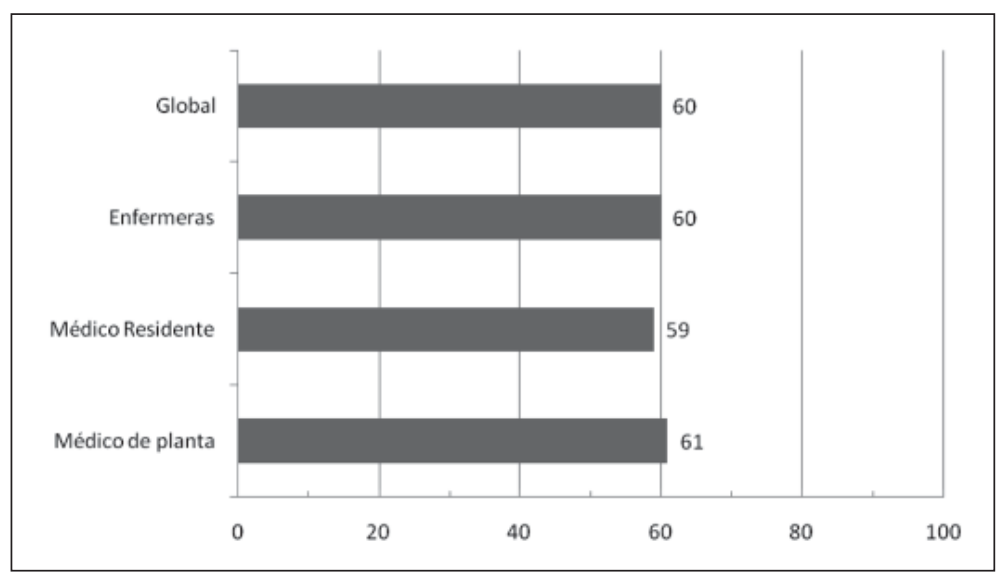

Figura 1. Porcentaje de respuestas correctas de los cuestionarios aplicados a cada estamento. No hubo diferencias significativas entre los grupos. ( $p: 0,93$ ). 
Hipoglicemia en adultos diabéticos hospitalizados - L. Rojas et al

Tabla 1. Porcentaje de respuestas correctas de cada estamento para cada ítem evaluado según el tipo de hipoglicemia

\begin{tabular}{|c|c|c|c|c|c|}
\hline & $\begin{array}{l}\text { Médicos de } \\
\text { planta }\end{array}$ & Residentes & Enfermeras & Global & Valor $\mathbf{p}$ \\
\hline \multicolumn{6}{|l|}{ Hipoglicemia asintomática } \\
\hline El manejo inicial & 72,7 & 85 & 71,4 & 76 & 0,306 \\
\hline Seguimiento precoz & 27,2 & 16,6 & 14,2 & 19,4 & 0,62 \\
\hline El valor de glicemia objetivo del manejo inicial & 45,4 & 42,8 & 60,7 & 49,6 & 0,329 \\
\hline $\begin{array}{l}\text { Monitorización posterior al logro de niveles } \\
\text { adecuados de glicemia }\end{array}$ & 27,2 & 23,8 & 57,1 & 36 & $0,014^{*}$ \\
\hline \multicolumn{6}{|l|}{ Hipoglicemia sintomática } \\
\hline El manejo inicial & 72,7 & 57,1 & 25 & 51,6 & $0,007^{*}$ \\
\hline Seguimiento precoz & 54,5 & 40,4 & 60,7 & 51,9 & 0,236 \\
\hline El valor de glicemia objetivo del manejo inicial & 45,4 & 42,8 & 60,7 & 49,6 & 0,329 \\
\hline $\begin{array}{l}\text { Monitorización posterior al logro de niveles } \\
\text { adecuados de glicemia }\end{array}$ & 27,2 & 23,8 & 57,1 & 36 & $0,014^{*}$ \\
\hline \multicolumn{6}{|l|}{ Hipoglicemia severa (paciente Inconsciente) } \\
\hline El manejo inicial & 90,9 & 85,7 & 78,5 & 85 & 0,579 \\
\hline Seguimiento precoz & 100 & 97,6 & 96,4 & 98 & 0,81 \\
\hline El valor de glicemia objetivo del manejo inicial & 27,2 & 42,8 & 32,1 & 34 & 0,51 \\
\hline $\begin{array}{l}\text { Monitorización posterior al logro de niveles } \\
\text { adecuados de glicemia }\end{array}$ & 36,3 & 26,1 & 35,7 & 32,7 & 0,639 \\
\hline
\end{tabular}

(p: significancia estadística).

los médicos de planta (30\%) y residentes $(24,5 \%)$ los que presentaron peores resultados (enfermeras $50 \%)$. Fueron también las preguntas relacionadas al seguimiento de las hipoglicemias con compromiso de conciencia las que generaron menor porcentaje de respuestas correctas. Se encontraron diferencias estadísticamente significativas entre los grupos sólo al evaluar el monitoreo de las hipoglicemias sintomáticas (Tabla 1).

\section{Discusión}

Los tres estamentos mostraron un buen nivel de conocimientos en el diagnóstico de la hipoglicemia. Sin embargo, este no ocurre al momento de definir su severidad, lo que cobra relevancia al definir el enfrentamiento terapéutico.

El manejo inicial fue el área mejor respondida por todos los encuestados y fue el único escenario donde se encontró diferencia entre los grupos (mejor en los médicos que en las enfermeras). Probablemente se deba a que son los médicos los que comúnmente asumen labores terapéuticas en la práctica clínica local. Sin embargo, creemos que tanto el diagnóstico como el tratamiento inicial de la hipoglicemia es responsabilidad de todos los estamentos que están en contacto directo con los pacientes, al ser una complicación que requiere de un manejo inmediato.

En este mismo escenario, fue en el grupo de pacientes con hipoglicemia con compromiso de conciencia donde se concentró el mayor porcentaje de respuestas correctas, a pesar de no conocer el valor glicémico que generalmente las genera (glicemia capilar bajo $40 \mathrm{mg} / \mathrm{dl}$ ). Esto podría explicarse porque en la práctica el manejo se ejecuta en forma similar para los distintos tipos de hipoglicemia, sin hacer diferencias de acuerdo a la severidad de éstas. Esta situación se traduce en la administración de glucosa por vía endovenosa o uso de glucagón en pacientes que se podrían tratar sólo con agua 
y azúcar, esto lleva a grandes variaciones de la glicemia y uso innecesario de recursos.

El seguimiento posterior es crucial para evitar las recurrencias, en especial cuando la mayoría de las causas que la generan (insulinas, hipoglicemiantes orales ya administrados, edad avanzada, falla renal o baja ingesta) no pueden ser modificados. Fue en este escenario donde se encontraron más errores, en especial en la hipoglicemia sin compromiso de conciencia y en el seguimiento tardío, es decir, después de haber obtenido un buen resultado inicial. Esto es especialmente relevante ya que este es el período donde más recurrencias se producen.

Estos resultados ponen de manifiesto un insuficiente conocimiento del equipo profesional hospitalario sobre las definiciones teóricas y las conductas clínicas sugeridas por la literatura para el enfrentamiento de los episodios hipoglicémicos en pacientes adultos hospitalizados. Esto adquiere especial relevancia debido al probable impacto en la morbimortalidad y costos asociados a la ocurrencia de la hipoglicemia y a la creciente difusión e implementación de estrictos esquemas de insulinoterapia intensificada.

Existen escasas publicaciones respecto a protocolos de manejo de hipoglicemia en pacientes hospitalizados. Esto podría deberse a la poca importancia que se le da a esta complicación, a una formación deficiente al respecto tanto en pre como en postgrado o a la sensación que el manejo es conocido por el equipo de salud.

Por lo anterior, creemos que se deben realizar intervenciones educativas para la prevención de la hipoglicemia mediante la identificación de los pacientes con mayor riesgo y la implementación de protocolos institucionales para el mejor manejo de esta complicación.

La implementación de protocolos ha permitido terapias más rápidas y eficientes que pueden ser aplicadas por cualquier miembro del equipo de salud (enfermeras, médicos o paramédicos). Sin embargo, su existencia no asegura un mejor manejo institucional de esta patología ya que existe el riesgo de baja adherencia a ellos ${ }^{25}$. Es fundamental entonces velar no sólo por la existencia de un protocolo de manejo, sino por una implementación activa de éste que asegure su uso en forma continua.

Cabe destacar que los algoritmos de enfrentamiento deben ser complementados con conductas para prevenir la ocurrencia, tales como identificar a pacientes de riesgo, el uso de protocolos de insulinoterapia que consideren esquemas de insulina basal prandial y el uso de hojas de indicaciones claras y legibles ${ }^{26}$, así como también deben incluir conductas para prevenir la recurrencia, tales como investigar la causa (baja ingesta, error en la administración, resolución de la causa de la hiperglicemia, etc.) y ajuste de las dosis de insulina ${ }^{13}$.

\section{Referencias}

1. Van den Berghe G, Wouters P, Weekers F, Verwaest C, Bruyninckx F, Schetz M, et al. Intensive insulin therapy in the critically ill patients. N Engl J Med 2001; 345: 1359-67.

2. Malmberg K, Norhammar A, Wedel H, Rydén L. Glycometabolic state at admission: important risk marker of mortality in conventionally treated patients with diabetes mellitus and acute myocardial infarction: long-term results from the Diabetes and Insulin-Glucose Infusion in Acute Myocardial Infarction (DIGAMI) study. Circulation 1999; 99: 2626-32.

3. Furnary AP, Zerr KJ, Grunkemeier GL, Starr A. Continuous intravenous insulin infusion reduces the incidence of deep sternal wound infection in diabetic patients after cardiac surgical procedures. Ann Thorac Surg 1999; 67: 352-60.

4. Baker EH, Janaway CH, Philips BJ, Brennan AL, Baines DL, Wood DM, et al. Hyperglycaemia is associated with poor outcomes in patients admitted to hospital with acute exacerbations of chronic obstructive pulmonary disease. Thorax 2006; 61: 284-9.

5. Cheung NW, Napier B, Zaccaria C, Fletcher JP. Hyperglycemia is associated with adverse outcomes in patients receiving total parenteral nutrition. Diabetes Care 2005; 28: 2367-71.

6. Frontera JA, Fernández A, Claassen J, Schmidt M, Schumacher HC, Wartenberg K, et al. Hyperglycemia after SAH: predictors, associated complications, and impact on outcome. Stroke 2006; 37: 199-203.

7. Clement S, Braithwaite SS, Magee MF, Ahmann A, Smith EP, Schafer RG, et al; American Diabetes Association Diabetes in Hospitals Writing Committee. Management of diabetes and hyperglycemia in hospitals. Diabetes Care. 2004; 27: 553-91.

8. Turchin A, Matheny ME, Shubina M, Scanlon JV, Greenwood B, Pendergrass ML. Hypoglycemia and Clinical Outcomes in Patients With Diabetes Hospitalized in the General Ward. Diabetes Care 2009; 32: 1153-7.

9. Cryer PE, Davis SN, Shamoon H. Hypoglycemia in dia- 
betes. Diabetes Care 2003; 26: 1902-12.

10. Shilo S, Berezovsky S, Friedlander Y, Sonnenblick M. Hypoglycemia in hospitalized nodiabetic older patient. J Am Geriatr Soc 1998; 46: 978-82.

11. Mortensen EM, García S, Leykum L, Nakashima B, Restrepo MI, Anzueto A. Association of hypoglycemia with mortality for subjects hospitalized with pneumonia. Am J Med Sci 2010; 339: 239-43.

12. Kagansky N, Levy S, Rimon E, Cojocaru L, Fridman A, Ozer Z, et al. Hypoglycemia as a predictor of mortality in hospitalized elderly patients. Arch Intern Med 2003; 163: 1825-9.

13. Tomky D. Detection, prevention and treatment of hypoglycemia in the hospital. Diabetes Spectr 2005; 18: 39-44.

14. Fischer KF, Lee JA, Newman JH. Hypoglycemia in hospitalized patients: cause and outcomes. N Engl J Med 1986; 315: 1245-50.

15. Maynard GA, Huynh M, Renvall M. Iatrogenic Inpatient Hypoglycemia: Risk Factors, Treatment, and Prevention: Analysis of Current Practice at an Academic Medical Center With Implications for Improvement Efforts. Diabetes Spectr. 2008; 21: 241-247.

16. Varghese P, Gleason V, Sorokin R, Senholzi C, Jabbour S, Gottlieb JE: Hypoglycemia in hospitalized patients treated with antihyperglycemic agents. J Hosp Med 2007; 2: 234-40.

17. Smith WD, Winterstein AG, Johns T, Rosenberg E, Sauer BC. Causes of hyperglycemia and hypoglycemia in adult inpatients. Am J Health Syst Pharm 2005; 62: 714-9.

18. "High-alert" medications and patient safety. Int J Qual Health Care 2001; 4: 339-40.

19. Kosiborod M, Inzucchi SE, Goyal A, Krumholz HM,
Masoudi FA, Xiao L, et al. Relationship between spontaneous and iatrogenic hypoglycemia and mortality in patients hospitalized with acute myocardial infarction. JAMA 2009; 301: 1556-64.

20. Van den Berghe G, Wilmer A, Hermans G, Meersseman W, Wouters PJ, Milants I, et al. Intensive insulin therapy in the medical ICU. N Engl J Med 2006; 354: 449-61.

21. Franz MJ, Kulkarni K, Polonsky WH, Yarborough PC, ZamudioV. A Core Curriculum for Diabetes Education. Eds. Diabetes Education and Program Management 4th ed. Chicago, Ill. American Association of Diabetes Educators; 2003.p. 3-18.

22. Garg R, Bhutani H, Jarry A, Pendergrass M: Provider response to insulin-induced hypoglycemia in hospitalized patients. J Hosp Med 2007; 2: 258-60.

23. Dinardo M, Korytkowski M, Calabrese A. Introduction of an inpatient hypoglycemia treatment protocol: effects on nurses' confidence and knowledge. Diabetes 2002; 51 (suppl 2): A212.

24. Moghissi ES, Korytkowski MT, DiNardo M; American Association of Clinical Endocrinologists. American Association of Clinical Endocrinologists and American Diabetes Association. Consensus statement on inpatient glycemic control. Diabetes Care 2009; 32: 1119-31.

25. Anthony M. Treatment of hypoglycemia in hospitalized adults: a descriptive study. Diabetes Educ 2007; 33: 70915.

26. Maynard G, Lee J, Phillips G, Fink E, Renvall M. Improved inpatient use of basal insulin, reduced hypoglycemia, and improved glycemic control: effect of structured subcutaneous insulin orders and an insulin management algorithm. J Hosp Med 2009; 4: 3-15.

\section{Anexo 1. Encuesta acerca de conocimientos en el diagnótico y manejo de la hipoglicemia en pacientes diabéticos hospitalizados}

1. Según la American Diabetes Association (ADA), existe hipoglicemia cuando la glicemia capilar (o hemoglucotest) es menor de :
a. $90 \mathrm{mg} / \mathrm{dl}$
b. $75 \mathrm{mg} / \mathrm{dl}$
c. $70 \mathrm{mg} / \mathrm{dl}$
d. $80 \mathrm{mg} / \mathrm{dl}$

2. El término hipoglicemia severa se define cuando la glicemia capilar es menor de:
a. $40 \mathrm{mg} / \mathrm{dl}$
b. $60 \mathrm{mg} / \mathrm{dl}$
c. $70 \mathrm{mg} / \mathrm{dl}$
d. $50 \mathrm{mg} / \mathrm{dl}$ 
3. En un paciente consciente con hipoglicemia sintomática, el primer paso para manejarlo es:
a. Dar alimentos vía oral, de acuerdo a su régimen alimenticio.
b. Dar un vaso de agua mezclado con 2 cucharadas sopera de azúcar, el cual debe ingerir en su totalidad.
c. Administrar 1 ampolla de suero glucosado al 30\% endovenosa $(20 \mathrm{cc})$ por vía periférica o central.
d. Administrar un suero glucosado al $10 \%$ a goteo rápido endovenoso por vía periférica o central.

4. El primer control de glicemia capilar después de haber manejado a un paciente conciente con hipoglicemia sintomática debe realizarse a los:
a. 5 minutos.
b. 15 minutos.
c. 30 minutos.
d. 60 minutos.

5. En un paciente consciente con hipoglicemia asintomática la medida a tomar es:
a. Dar alimentos vía oral, de acuerdo a su régimen alimenticio, ya sea una colación o adelantar su próxima comida.
b. Dar un vaso de agua mezclado con 2 cucharadas sopera de azúcar, el cual debe ingerir en su totalidad.
c. Administrar 1 ampolla de suero glucosado al $30 \%$ endovenosa $(20 \mathrm{cc})$ por vía periférica o central.
d. Administrar un suero glucosado al $10 \%$ a goteo rápido endovenoso por vía periférica o central.

6. El primer control de glicemia capilar después de haber manejado a un paciente conciente con hipoglicemia asintomática debe realizarse a los:
a. 60 minutos.
b. 30 minutos.
c. 15 minutos.
d. 5 minutos.

7. El objetivo mínimo esperado de glicemia capilar una vez ejecutado el manejo inicial de hipoglicemia para paciente conciente sintomático o asintomático es:
a. $60 \mathrm{mg} / \mathrm{dl}$
b. $70 \mathrm{mg} / \mathrm{dl}$
c. $80 \mathrm{mg} / \mathrm{dl}$
d. $90 \mathrm{mg} / \mathrm{dl}$

8. Después de alcanzar un nivel adecuado de glicemia en el primero control glicémico, en paciente conciente sintomático, el (los) siguiente (s) control (es) de glicemia deberán realizarse:
a. Cada 1 hora hasta obtener 2 valores euglicémicos consecutivos.
b. Cada 30 minutos hasta obtener 2 valores euglicémicos consecutivos.
c. Continuar con los controles habituales.
d. Cada 30 minutos hasta obtener 2 valores euglicémicos consecutivos.

9. En un paciente inconsciente secundario a una hipoglicemia severa, la primera medida que Usted tomaría es:
a. Llamar al médico tratante.
b. Manejar al paciente: evaluar presencia o ausencia de vía venosa y de acuerdo a eso definir el manejo.
c. Trasladar a unidad de mayor complejidad.
d. Tomar examen de sangre (glicemia).

10. El tratamiento medicamentoso que usted utilizaría para un paciente inconsciente con hipoglicemia sería:
a. Administrar suero glucosado al $10 \%$ a goteo rápido por vía venosa periférica o central.
b. Administrar 4 ampollas de suero glucosado al $30 \%$ por vía venosa periférica o central.
c. Administrar suero glucosado al $20 \%$ por vía venosa periférica.
d. Administrar 1 ampolla de suero glucosado al 30\% intramuscular. 
Hipoglicemia en adultos diabéticos hospitalizados - L. Rojas et al

11. El primer control de glicemia capilar después de haber manejado a un paciente inconsciente con hipoglicemia y que no responde debe realizarse a los:
a. 15 minutos.
b. 10 minutos.
c. 25 minutos.
d. 30 minutos.

12. El valor mínimo esperado de glicemia capilar una vez ejecutado el manejo inicial de hipoglicemia para paciente inconsciente es:
a. $60 \mathrm{mg} / \mathrm{dl}$
b. $70 \mathrm{mg} / \mathrm{dl}$
c. $80 \mathrm{mg} / \mathrm{dl}$
d. $90 \mathrm{mg} / \mathrm{dl}$

13. Si a pesar del manejo inicial el paciente inconsciente sigue hipoglicémico la conducta a seguir es:
a. Repetir el tratamiento medicamentoso que utilizó como manejo inicial.
b. Administrar 1 ampolla de suero glucosado al 5\% endovenosa.
c. Administrar 1 ampolla de suero glucosado al $30 \%$ endovenosa.
d. Colocar suero glucosado al 10\% a $100 \mathrm{ml} /$ hora. 